\title{
Traduire
}

Une eutre perspective sur $r$ tatadciction

Revue française de la traduction

245 | 2021

Regards sur l'interprétation

\section{Co-interprétation sourd et entendant. L'union fait la force}

Nicolas Hanquet et Delphine le Maire

\section{(2) OpenEdition}

1 Journals

Édition électronique

URL : https://journals.openedition.org/traduire/2470

DOI : 10.4000/traduire.2470

ISSN : 2272-9992

Éditeur

Société française des traducteurs

Édition imprimée

Date de publication : 5 décembre 2021

Pagination : 86-98

ISSN : 0395-773X

\section{Référence électronique}

Nicolas Hanquet et Delphine le Maire, « Co-interprétation sourd et entendant. L'union fait la force »,

Traduire [En ligne], 245 | 2021, mis en ligne le 05 décembre 2021, consulté le 23 décembre 2021. URL :

http://journals.openedition.org/traduire/2470 ; DOI : https://doi.org/10.4000/traduire.2470 


\section{Co-interprétation sourd et entendant}

L'union fait la force

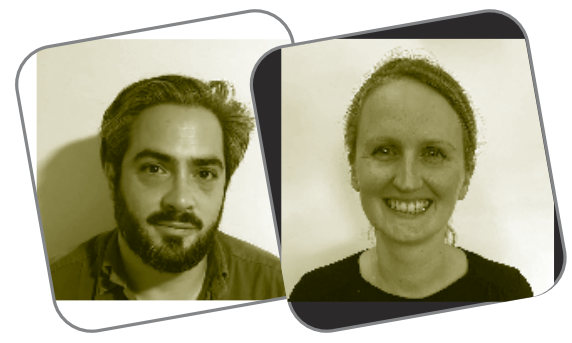

Nicolas Hanquet
Delphine le Maire

\section{Introduction}

Au début de la crise sanitaire, le 17 mars 2020, l'État belge décide de rendre accessibles à la communauté des Sourds ${ }^{1}$ les conférences de presse sur l'évolution de la situation épidémiologique liée au SRAS-CoV-2 (Covid-19). Le dispositif mis en place s'appelle la co-interprétation, processus spécifique d'interprétation simultanée réalisée par un interprète entendant «pivot» et un interprète sourd «relais». Cette technique vise à la production d'une interprétation au plus proche d'une langue naturelle. C'est la première fois que, d'une part, ce dispositif est utilisé dans les médias belges et que, d'autre part, deux équipes travaillent consécutivement à fournir de l'interprétation en langue des signes de Belgique francophone et en langue des signes flamande. Cette pratique, bien que largement répandue aux États-Unis et au Canada, fait ses premiers

1. L'emploi de la majuscule à «Sourds» permet d'identifier ici les sourds en tant qu'individus d'une communauté linguistique minoritaire se réclamant d'une identité culturelle spécifique. 
pas en Europe. Nous souhaitons, par ce partage d'expérience, promouvoir son développement dans divers domaines. Nous vous proposons ici, après une mise en contexte, un récit de l'instauration de cette technique d'interprétation agrémenté de liens vers divers modèles théoriques de l'interprétation ainsi que des réflexions basées sur la pratique.

\section{Le contexte belge}

La Belgique est un pays fédéral dont la structure politique est complexe: elle est composée de trois régions (Région wallonne, Région de Bruxelles-Capitale et Région flamande) et de trois communautés linguistiques (Communauté française, Communauté germanophone et Communauté flamande). Les langues officielles reconnues au niveau fédéral sont le français, le néerlandais et l'allemand. Les trois langues des signes, à savoir la langue des signes de Belgique francophone (LSFB), la langue des signes flamande (Vlaamse Gebarentaal, VGT) et la langue des signes allemande (Deutsche Gebärdensprache, DGS) sont, quant à elles, reconnues symboliquement au sein de leur communauté respective. Nous ne disposons pas actuellement de données exactes concernant le nombre d'utilisateurs des trois langues des signes en Belgique. Cependant, la Fédération francophone des Sourds de Belgique estimait en 2019 qu'ils étaient entre 30000 et 50000 .

En Belgique francophone, les acteurs de l'interprétation sont soit entendants soit sourds. Les interprètes françaisLSFB sont rares à l'heure actuelle en Communauté française et, si l'on compare leur nombre à celui des utilisateurs pouvant faire appel à leurs services, le déséquilibre est important. Les raisons de cette pénurie sont multiples; parmi elles, on compte, entre autres, l'ouverture tardive d'une formation de niveau universitaire, qui n'a vu le jour qu'en 2014 à I'Institut libre Marie-Haps (aujourd'hui Université SaintLouis à Bruxelles) pour les trois premières années du cursus et à l'Université catholique de Louvain (UCLouvain) pour les deux dernières. Les premiers interprètes diplômés en sont sortis en 2019. En trois ans, huit étudiants ont obtenu leur diplôme. Ces études permettent l'apprentissage de l'interprétation du français oral vers la LSFB et inversement. 
L'UCLouvain propose également une formation en traduction de la LSFB vers le français écrit. Diverses formations courtes ont permis la certification d'interprètes avant 2014, portant aujourd'hui le nombre de ces professionnels à une trentaine. Les interprètes français-LSFB travaillent dans des contextes variés (médical, juridique, scolaire, professionnel, etc.) : la demande dépassant de loin les ressources humaines, ils n'ont d'autre choix que de diversifier leur pratique.

Quant aux interprètes sourds, il est difficile de les dénombrer en raison des contextes divers dans lesquels ils travaillent et de l'absence d'une reconnaissance officielle de leur profession. Ils se distinguent des interprètes français-LSFB par leur appartenance culturelle à la communauté des Sourds et leur bilinguisme, et se spécialisent dans "l'adaptation culturelle et linguistique du message à transmettre» (Carlier et al., 2016). Leurs compétences peuvent varier de la traduction à l'interprétation entre plusieurs langues (écrites ou signées) sans oublier l'utilisation de codes de communication adaptés aux personnes sourdes-aveugles, et ce, seul ou en collaboration avec un interprète français-LSFB entendant. Les demandes d'intervention des interprètes sourds varient selon les besoins de la communauté sourde: interprétation de liaison (par exemple, dans le domaine de la santé mentale ou de la migration), interprétation de conférences, traduction de textes français vers la LSFB, etc. À ce jour, aucun cursus long ne leur est accessible, et le master en interprétation de I'UCLouvain ne fait pas exception. Néanmoins, certaines formations courtes leur sont ouvertes, comme le certificat universitaire en interprétation en contexte juridique (milieu judiciaire et secteur des demandes d'asile) de l'Université de Mons, obtenu par cing interprètes sourds.

\section{La co-interprétation: quelques notions clés pour comprendre son fonctionnement}

Les stratégies à disposition des interprètes en langue vocale ont été, et sont toujours, largement étudiées ${ }^{2}$.

2. Les concepts théoriques sont abordés en guise d'illustration de la logique qui soustend l'exercice de co-interprétation, nous ne les développons que superficiellement. 
Les travaux de Seleskovitch et Lederer (1984) restent fondateurs jusqu'à aujourd'hui. La déverbalisation (Seleskovitch et Lederer, 1984) permet le détachement du sens du discours de la langue de départ pour permettre sa reformulation dans la langue d'arrivée. Le skopos (Vermeer et Reiss, 1984) est la prise en compte de la fonction spécifique de toute traduction et l'adaptation à son public cible. L'écart linguistique (Seleskovitch et Lederer, 1984) représente l'ensemble des différences culturelles et de représentations du monde entre deux langues. À ces trois concepts théoriques s'ajoute la théorie des efforts (Gile, 1985) qui explique la combinaison des efforts d'écoute, de déverbalisation et de reformulation, ainsi que l'effort de maintien de l'équilibre entre ces trois premiers efforts, que l'interprète en action réalise simultanément. Cette théorie, appliquée à l'interprétation en langue des signes (Pointurier, 2014), décrit les processus cognitifs nécessaires à l'exercice de l'interprétation simultanée, dont, notamment, l'effort supplémentaire de mémorisation des emplacements et des pointages en langue des signes ${ }^{3}$. L'interprétation vers une deuxième langue dont la complexité est renforcée par l'écart significatif entre une langue audiophonatoire et une langue visuo-gestuelle est un exercice hautement exigeant (Pointurier, 2014; Bélanger, 1998).

La représentation du monde diffère grandement entre une personne qui entend et une personne qui appréhende le monde uniquement à travers ses yeux (Delaporte, 2002). Pour les interprètes entendants, apprendre à percevoir le monde sous un angle inconnu et presque étranger exige une formation intensive ainsi que de longues années de rencontres et de pratique de la langue. Outre la nécessaire maîtrise de ses langues de travail, l'interprète dispose de stratégies spécifiques à l'exercice de sa fonction. Pour préserver l'équilibre entre les efforts nécessaires à l'interprétation simultanée, il peut choisir d'omettre certaines parties du discours (Napier, 2004), telles que les informations secondaires, les répétitions, les hésitations, de passer d'une forme de discours direct à un

3. L'utilisation de l'espace est spécifique en langue des signes. L'espace de signation tridimensionnel est investi par le locuteur, il est également utilisé comme marqueur pronominal, entre autres par l'utilisation du pointage ou des mouvements du regard (Meurant, 2008). 
discours indirect, et inversement, d'utiliser l'exemplification ou la généralisation (Bélanger, 1998), etc.

L'emploi de ces stratégies et l'importante charge cognitive de l'interprétation vers une deuxième langue peuvent altérer la qualité du discours dans la langue d'arrivée (Bernard, 2007), qualité pâtissant déjà du fait que, contrairement aux interprètes des langues vocales qui travaillent vers leur langue maternelle, la plupart des interprètes en langue des signes travaillent vers une langue seconde, apprise souvent tardivement (Humphrey, 1995; Boudreault, 2005). Si l'on ajoute à cela la diversité des variantes régionales d'une même langue des signes mais aussi la diversité des profils linguistiques parmi les Sourds signants, on comprend alors aisément pourquoi les interprètes entendants sont régulièrement amenés à collaborer avec les interprètes sourds.

La co-interprétation peut être abordée au travers de différents modèles théoriques. Les pratiques auxquelles le terme fait allusion varient selon les auteurs et les contextes, avec pour dénominateur commun l'interprétation en binôme, au minimum. La co-interprétation peut soit être un processus dit «intra-lingual» (Nassa Gassa Gonga, 2020) lorsque l'interprète sourd reformule une même langue des signes au départ du feeding ${ }^{4}$ de l'interprète entendant, soit s'appliquer à l'interprétation en relais, par exemple lorsqu'une langue intermédiaire est utilisée pour arriver à la langue d'arrivée. C'est une pratique largement répandue dans les conférences internationales (ibid.).

De Meulder et Heyerick (2013) proposent une liste d'arguments en faveur des interprètes sourds, à partir de laquelle nous avons construit notre proposition pour la cointerprétation des conférences de presse. En guise d'illustration, voici deux arguments qui mettent en lumière, selon nous, l'importance de la collaboration entre interprètes sourds et entendants:

- La dimension culturelle: les interprètes sourds sont membres de la communauté pour laquelle ils travaillent. Les interprètes sourds compétents ont la capacité de

4. Terme largement employé dans la littérature anglaise pour désigner le travail de transmission du discours interprété par l'interprète pivot (Ressler, 1999; Stone et Russell, 2014). 
penser de la même manière que les autres Sourds, s'appuyant sur une expérience du monde acquise par le regard et sur sa conceptualisation grâce au visuel (Stone, 2009). Ils sont déjà adaptés à l'esprit des spectateurs (Duncan, 1997). Cette appartenance commune et cette identification culturelle sont des facteurs importants pour établir un rapport effectif de communication (Boudreault, 2005).

- La dimension linguistique: les interprètes sourds expriment une perspective intérieure, montrant à voir des actions construites ou des dialogues dans lesquels ils sont eux-mêmes impliqués, et non pas un point de vue extérieur aux situations décrites (Stone, 2009).

La recherche montre l'intérêt certain de cette collaboration. C'est seulement au regard de la complémentarité des compétences individuelles et spécifiques que la cointerprétation prend tout son sens. Ce n'est pas parce que l'interprète entendant n'est pas suffisamment compétent que nous avons développé cette technique, mais bien parce que le travail d'équipe offre une vraie plus-value et qu'il permet également aux citoyens sourds d'être les acteurs centraux des services qui les concernent.

\section{L'origine de la demande}

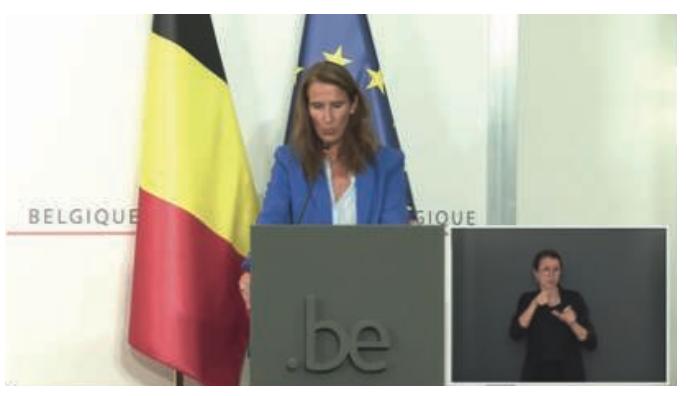

Aperçu public de la co-interprétation d'une conférence de presse donnée par la Première Ministre Sophie Wilmès. @ Chaîne Youtube de la Chancellerie de la Première Ministre.

Le Centre de crise national (CCN) a d'abord approché des interprètes entendants, en Flandre et en Wallonie. Après de premiers échanges, nous avons pris contact avec nos collègues sourds pour construire une proposition. En amont, les fédérations des Sourds (FFSB/Doof Vlanderen) avaient déjà sensibilisé le CCN à la nécessité de faire appel à des locuteurs sourds en cas de communication de crise et les deux fédérations ont soutenu le projet de co-interprétation que nous souhaitions mettre en place (Gebruers et Haesenne, 
à paraître). Le CCN a accordé son soutien pour une information interprétée en langue des signes par des locuteurs sourds pour le public sourd varié (Gebruers et al., à paraître). La demande initiale était d'interpréter des conférences de presse pendant deux semaines. Ces conférences ont finalement été données pendant presque une année et demie et leur nombre s'élève à plus de $200^{5}$.

\section{Comment ça marche?}

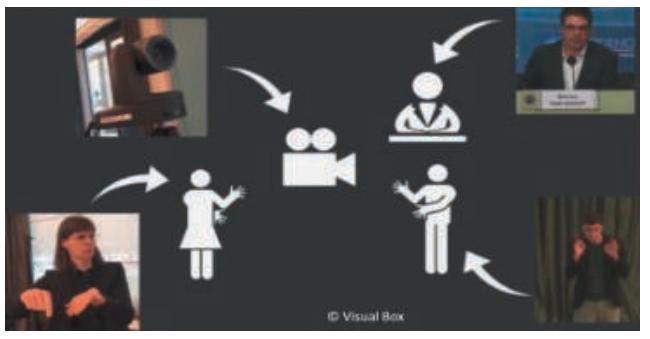

Schéma interne de la co-interprétation lors d'une conférence de presse. (c) Avec l'aimable accord de VisualBox.

L'interprète entendant «pivot» est situé à côté de la caméra et interprète le discours des orateurs francophones ou néerlandophones. Il fait face à l'interprète sourd «relais», qui se place face à la caméra. L'interprète pivot lui transmet une version épurée du discours interprété en LSFB ou en VGT. L'interprète sourd reformule simultanément le discours pour les téléspectateurs sourds. Notons que les textes des orateurs sont transmis en amont aux équipes d'interprètes, la grande majorité du discours est dès lors connue et préparée avant la conférence. Les chiffres liés aux contaminations, hospitalisations et décès sont nombreux, ils sont préalablement indiqués sur un tableau sous la caméra.

Ce qui nous est apparu comme l'avantage le plus considérable de cette technique est la répartition des efforts d'interprétation entre les membres du binôme, chacun étant également responsable de la production dans la langue d'arrivée. Cette répartition permet, d'une part, à l'interprète entendant de se concentrer davantage sur le contenu du discours à traduire et, d'autre part, à l'interprète sourd d'avoir plus d'énergie pour rendre une langue des signes au plus proche d'une expression naturelle, évitant tant que faire se peut la dégradation de la production inhérente aux efforts 
cognitifs à fournir. Notons ici que l'interprétation dans les médias est à destination de l'ensemble de la communauté des Sourds; il est dès lors impossible de s'adapter à un profil linguistique spécifique. L'effort supplémentaire que représente l'adaptation à un large public diversifié nécessite,

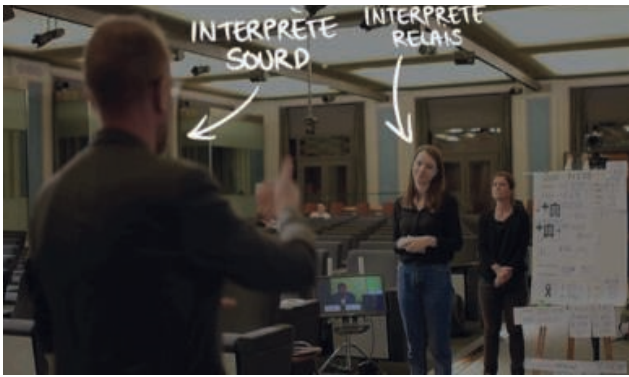

Exemple de chiffres écrits au préalable sur un tableau en guise de support pour l'interprète sourd. (c) Image prise par Julie Carlier (MUSK). pour nous, le recours à la co-interprétation.

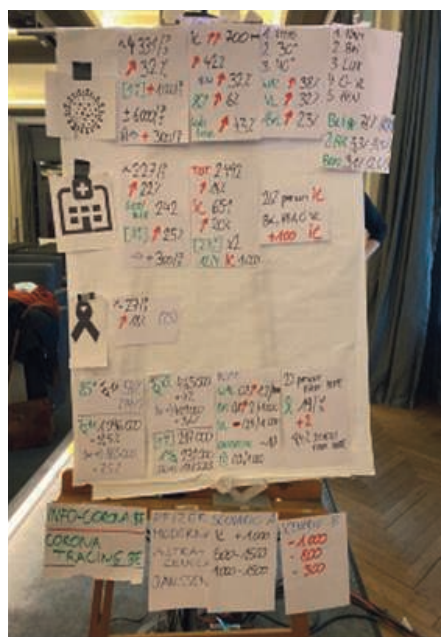

Aperçu de la composition de l'équipe d'interprètes réalisant le travail de co-interprétation. ( ) Image issue du documentaire de MUSK.

Pour en savoir plus sur la $\mathrm{co}^{-}$ interprétation dans le contexte présenté, nous vous invitons à consulter notre présentation donnée en collaboration avec nos collègues lors de la conférence internationale de la World Association of Sign Language Interpreters (WASLI) ${ }^{6}$, le documentaire produit par nos partenaires MUSK et Cosens ${ }^{7}$ ainsi que l'interview réalisée par VisualBox ${ }^{8}$.

\section{Quelques réflexions sur notre expérience}

Outre les liens avec la recherche, cette expérience a été riche en enseignements pour chacun et chacune d'entre nous. Nous avons souhaité aborder ici quelques éléments 
plus subjectifs, propres à nos deux expériences spécifiques lors de l'interprétation des conférences de presse.

Étant donné que nous, interprètes entendants «pivot» et interprètes sourds «relais», collaborons aussi bien au niveau de la préparation que de l'interprétation, sans oublier l'évaluation des prestations, nous avons pu partager des connaissances et des savoir-faire issus de nos langues maternelles et de nos cultures respectives, créant ainsi un pont culturel entre nous. Outre les prestations en tant que telles, ces échanges quotidiens sont des occasions sans cesse renouvelées de nourrir nos compétences respectives.

Les interprètes "pivots» ont rapidement constaté des différences notables entre une interprétation directe et la cointerprétation. La recherche sur cette question étant encore balbutiante, il est encore difficile de nommer précisément ces différences. Elles résident probablement dans des modifications grammaticales et syntaxiques spécifiques en vue de libérer de l'espace pour que les interprètes sourds puissent redessiner plus adéquatement le discours depuis leur propre perspective. II ne s'agit pas pour autant d'omettre des pans importants de discours, mais d'épurer la langue jusqu'à un certain point, de la rendre plus «lisse» pour en faciliter sa réception et sa reformulation. La préparation en amont jove un rôle fondamental, cela nous permet d'avancer sur un terrain relativement connu pour tous les interprètes. La charge cognitive est répartie sur une équipe et non prise en charge uniquement par un seul professionnel. La fréquence quotidienne des conférences de presse, interprétées par une petite équipe d'interprètes, a également été fondamentale dans le développement de cette pratique, chaque jour apportant son lot d'obstacles à résoudre et d'observations partagées.

En guise d'illustration, nous avons choisi de développer deux contraintes d'interprétation particulières entre langue orale et langue des signes.

Les interprètes, s'accordant un temps pour déverbaliser le discours, doivent sans cesse gérer un décalage entre ce qu'ils entendent et ce qu'ils produisent (Gile, 1985). Ce temps, variable en fonction du contenu du discours, est de l'ordre de quelques secondes. Lorsque l'interprète travaille seul, il est amené à allonger et à réduire ce temps avec une certaine liberté. Le travail de co-interprétation a amené les interprètes 
à gérer ce décalage différemment. D'une part, les interprètes entendants ont dû le réduire pour éviter un allongement trop important résultant du truchement de l'interprétation en binôme et permettre aux interprètes entendants de passer la main lors du basculement du français au néerlandais et inversement. D'autre part, pour permettre aux interprètes sourds de développer leur production en langue des signes, les interprètes entendants ont également dû apprendre à manier cet effort de mémoire avec beaucoup de souplesse. La saturation cognitive qui en découle peut entraîner des pertes d'information; c'est pour cette raison que les interprètes entendants travaillent à deux, le binôme de soutien étant chargé d'ajouter les informations omises en langue des signes auprès de l'interprète sourd, qui a lui-même dû développer des capacités spécifiques en vue de recevoir plusieurs pans de discours consécutivement.

Enfin, tout interprète s'est déjà heurté à la difficulté que représente l'interprétation simultanée de métaphores et d'expressions idiomatiques. Même si les conférences de presse avaient pour objectifs de vulgariser les découvertes sur la Covid-19 et d'informer la population sur les mesures de protection à suivre, elles n'en étaient pas moins riches en images et métaphores. Chemin faisant, nous avons dû utiliser de nombreuses périphrases et des structures de grande iconicité (Cuxac, 2000) pour expliciter visuellement certains concepts culturels et scientifiques. Nous avons aussi dû adapter certains signes utilisés habituellement dans un espace en trois dimensions (3D) afin qu'ils soient reconnaissables pour les téléspectateurs sourds via un écran en deux dimensions (2D). De plus, grâce aux allers-retours entre les conférences de presse et la communauté des Sourds, le bagage linguistique lié à la Covid-19 s'est petit à petit élargi au sein de la LSFB et de la VGT.

\section{Conclusion}

Cette première expérience a été riche en enseignements. Grâce au soutien et à la confiance du CCN et du gouvernement belge, nous avons pu contribuer à une meilleure accessibilité de l'information de crise à destination des citoyens 
sourds ${ }^{9}$. II nous semble aujourd'hui fondamental de soutenir l'accessibilité des médias en langue des signes en partenariat avec et pour le public concerné, ce à quoi invite, par ailleurs, la Convention de l'Organisation des Nations unies relative aux droits des personnes handicapées ${ }^{10}$.

Nous avons également pu découvrir les nombreux avantages que représente le travail en équipe d'interprètes sourds et entendants. La complémentarité des uns et des autres, la relation essentielle de confiance que nous avons dû construire ensemble et les nombreux échanges ont fait de nous de meilleurs professionnels.

Le temps est venu pour nous de prendre du recul, d'analyser et d'étudier nos pratiques et de formaliser nos apprentissages pour les transmettre. Ainsi, un certificat universitaire vient de voir le jour à l'Université de Namur, en partenariat avec MUSK, service d'accessibilité en langue des signes qui dispose d'un réseau d'interprètes sourds, et avec Cosens Coop', coopérative d'interprètes entendants, invitant les étudiants sourds et entendants à se former à la co-interprétation. Outre la communication dite "de crise», nous souhaitons explorer d'autres secteurs, comme le théâtre, l'adaptation spécifique de médias pour enfants sourds, etc. Enfin, l'une des directions nécessaires pour le développement de cette technique sera de co-construire nos projets à venir en relation directe avec les bénéficiaires de nos futures interprétations.

\section{nicolas.hanquet@usaintlouis.be delphinelemaire@me.com}

Nicolas HANQUET est interprète entendant français-LSFB, diplômé d'un master en interprétation de I'UCLouvain. II mène depuis septembre 2021 une thèse de doctorat sur la cointerprétation en tant qu'aspirant FNRS au sein des universités de Saint-Louis à Bruxelles (USL-B) et de Namur (UNamur).

9. Pour preuve, depuis cette expérience, le CCN fait appel à l'équipe d'interprètes entendants et sourds pour la co-interprétation de conférences de presse dans le cadre d'autres crises, telles que les inondations survenues en Belgique à la mi-juillet 2021.

10. Voir articles $4 \S 3$ et 9 de la convention précitée. 
Delphine LE MAIRE est interprète sourde. Détentrice d'un master en droit à l'UCLouvain, elle a obtenu un certificat d'université en interprétation en contexte juridique à l'Université de Mons. Elle est chargée de cours dans le programme du master en interprétation de l'UCLouvain.

\section{SOURCES CITÉES}

BELANGER Danielle-Claude, Les spécificités de l'interprétation en langue des signes québécoise, Montréal, Université du Québec à Montréal, 1998, https://www. academia.edu/15987281/Les_spécificités_de_linterprétation_en_langue_des_ signes_québécoise, consulté le 25/08/2021.

BERNARD Alexandre et al., L'interprétation en langue des signes, Paris, Presses Universitaires de France, 2007.

BOUDREAULT Patrick, "Deaf interpreters", in Topics in Signed Language Interpreting, 63, 2005, p. 323-355.

CARLIER Julie et al., Interprète sourd : portrait de compétence, Bruxelles, Info-Sourds de Bruxelles, 2016.

CUXAC Christian, La langue des signes française (LSF): les voies de l'iconicité, Paris, Ophrys, 2000.

DE MEULDER Maartje et HEYERICK Isabelle, «(Deaf) Interpreters on Television: Challenging Power and Responsibility », in MEURANT Laurence et al. (dir.), Sign Language Research, Uses and Practices: Crossing Views on Theorical and Applied Sign Language Linguistics, Berlin, De Gruyter, 2013, p. 111-136.

DELAPORTE Yves, Les sourds c'est comme ça, Paris, Éditions de la Maison des sciences de l'homme, 2002.

DUNCAN Bob, «Deaf People Interpreting on Television », in Deaf Worlds, 13(3), 1997, p. 35-39.

ECO Umberto, Dire presque la même chose. Expériences de traduction, Paris, Éditions Grasset et Fasquelle, 2006.

Fédération francophone des Sourds de Belgique (FFSB), Rapport d'activités 2019, Bruxelles, Fédération francophone des Sourds de Belgique, 2019, www.ffsb.be/ ra2019/, consulté le 25/08/2021.

GEBRUERS Karolien et HAESENNE Thierry, «Providing Co-interpreting Teams of Deaf and Hearing Signed Language Interpreters at Belgian Covid19 Press Briefings: A Silver Lining? », in DE CLERCK Goedele (dir.), UNCRPD Implementation in Europe - a Deaf Perspective. Article 9: Access to Information and Communication, Bruxelles, European Union of the Deaf, à paraître. 
GEBRUERS Karolien et al., «"Finally it Was Time to Provide a Deaf Interpreter": A Multiparty Perspective on the Provision of Signed Language Interpreters During the Covid-19 Press Briefings in Belgium ", in FEDERICI Frederico et O'BRIEN Sharon (dir.), Translating Crisis, London, Bloomsbury Publishing Plc., à paraître.

GILE Daniel, «Le modèle d'efforts et l'équilibre d'interprétation en interprétation simultanée», in Meta, 30(1), 1985, p. 44-48.

HUMPHREY Janice et BOB Alcorn, So You Want To Be An Interpreter? An Introduction to Sign Language Interpreting, Amarillo (États-Unis), H \& H Publishers, 1995.

MEURANT Laurence, Le regard en langue des signes. Anaphore en langue des signes française de Belgique (LSFB): morphologie, syntaxe, énonciation, Namur (Belgique), Presses universitaires de Namur, 2008.

NANA GASSA GONGA Aurélia, "Interpréter en signes internationaux: état des lieux en France et à l'international», in La main de Thôt, 7, 2020, http://revues. univ-tlse2.fr/lamaindethot/index.php?id=786, consulté le 25/08/2021.

NAPIER Jemina, «Interpreting Omissions, a New Perspective», in Interpreting. International Journal of Research and Practice in Interpreting, 6(2), 2004, p. 117-142.

POINTURIER Sophie, L'interprétation en langue des signes française: contraintes, tactiques, efforts, thèse, Paris, Université Sorbonne-Nouvelle, 2014.

RESSLER Carolyn, «A Comparative Analysis of a Direct Interpretation and an Intermediary Interpretation in American Sign Language», in Journal of Interpretation, 1999, p. 71-102.

SELESKOVITCH Danica et LEDERER Marianne, Interpréter pour traduire, Paris, Les Belles Lettres, 2014 (1984).

STONE Christopher, Towards a Deaf Translation Norm, Washington D.C., Gallaudet University Press, 2009.

STONE Christopher et RUSSELL Debra, "Conference Interpreting and Interpreting Teams », in ADAM Robert et al. (dir.), Deaf Interpreters at Work: International Insights, Washington D.C., Gallaudet University Press, 2014, p. 140-156.

VERMEER Hans et REISS Katharina, Towards a General Theory of Translational Action: Skopos Theory Explained, London and New York, Routledge, 2014 (1984). 